\title{
Managing Students' Learning through Reflection on Doing through a Self-Tutoring System
}

\author{
Yifan Zhou, Yanwei Sun, Zachary Ball, Zhenjun Ming, \\ Janet K. Allen, Farrokh Mistree \\ School of Computer Science and Technology, Beijing Institute of \\ technology, China/School of Mechanical Engineering, Beijing Institute \\ of technology, China/Mechanical Engineering Department, Carnegie \\ Mellon University, USA/School of Mechanical Engineering, Beijing \\ Institute of technology, China/Systems Realization Laboratory, \\ University of Oklahoma, USA/Systems Realization Laboratory, \\ University of Oklahoma, USA
}

\section{Keywords}

Engineering Design, Design Education, Learning Statements, Self-Tutoring System, student paper

\section{Extended Abstract}

How can students leverage the historical learning reflection data collected from former students in design, build, and test courses to tutor themselves in their own learning through reflection on doing?

University of Oklahoma (OU) offers a course named AME4163: Principles of Engineering Design. In the course, students learn by reflecting on doing in an immersive authentic environment[1]. After taking the course, the students need to write learning statements. A learning statement is a structured [Experience|Learning|Value] text-based construct for students in AME4163 Principles of Engineering Design to record what they learned by reflecting on authentic immersive experiences throughout the semester. The immersive experiences include lectures, assignments, reviews, building, testing and a post-analysis for design of an electromechanical system to address a given customer need. Over the past four years. at the University of Oklahoma, we have collected almost 32,000 learning statements from about 600 students. In this paper, we look at the data from the students' perspective and focus on demonstrating how students can utilize the former students' learning statements as a knowledge base to tutor themselves in their own learning through reflection on doing. We developed a self-tutoring system that allows students to compare their own learning statements to that of the former students in three ways, namely, Experience, Learning, and Value. The core of the self-tutoring system is a semantics-based similarity metric that enables the automatic searching of historical learning statements with similar experience, learning, or value at the semantic level.

To show how our self-tutoring system can benefit students, we use a subset of the learning statement database to build the self-tutoring system and input learning statements which are not in the subset. The results prove that students can learn from the self-tutoring system in different ways. When students entering a learning statement, they can get learning statements with similar 
experience, learning, or value. Students can analyze these learning statements and think about why other students have similar or different thoughts. They can also observe the similarity distribution diagrams which provide similarity information to other students visually.

By using our self-tutoring system, students can search former learning statements with similar experience, learning or value quickly, read these learning statements and expand their own learning statements. Although our self-tutoring system is designed for students, it is also helpful to other stakeholders. We summarize the benefits as follows:

1) Benefit to students: Students can get knowledge whenever and wherever they want. They can study without the help of teachers.

2) Benefit to instructors: The instructors can spend less time on assess students' learning statement because students can do this by themselves. The self-tutoring provides convenient methods to search learning statements, which make instructors access great learning statements easily and show them to students.

3) Benefit to researchers: We propose an ITS architecture based on SBERT which can explore the knowledge from all kinds of data. Researchers can replace the data in knowledge base to create a new ITS in different field.

Yifan Zhou - Earned a Bachelor of Engineering degree in Computer Science and Technology from Beijing Institute of Technology. He is now an algorithm researcher in Shanghai AI Lab. He is working on the deployment of Openmmlab. 